\title{
LETTER
}

\section{How did you sleep in the ICU?}

\author{
Laetitia Franck*, Jean-Pierre Tourtier, Nicolas Libert, Laurent Grasser and Yves Auroy
}

Sleep is important for healing and survival of critical illness, as far as quantitative and qualitative sleep deprivation can have negative consequences on a physiologic function, particularly the immune mechanism, as well as psychological well-being [1].

Patients' perception of intensive care is very different depending on the study. To illustrate, Simini reported frequent feelings such as 'pain, noise, sleep deprivation, thirst, hunger, fear, anxiety, and isolation' [2], but Granja and colleagues showed that $38 \%$ of patients did not remember at all their intensive care unit (ICU) stay and that $93 \%$ described the ICU environment as friendly and calm [3]. These authors also reported contrasting results: Simini reported that $61 \%$ of patients had sleep deprivation in the ICU, whereas sleep was described as being good and sufficient by $73 \%$ of patients in the study by Granja and colleagues.

We explored the memorization of sleep disturbances during an ICU stay and then evaluated the quality of sleep reported by patients after critical care.

A telephone interview including a random sample of 60 patients admitted to our ICU was performed 6 to 12 months after their discharge. Conventional intensive care variables were recorded from the ICU database and sleep disturbances were evaluated using the Basic Nordic Sleep Questionnaire [4]. Two nonvalidated questions were also analyzed: one estimated the quality of sleep in the ICU, while the other compared the quality of sleep before and after a stay in the ICU.

Among 53 responding patients, 47\% remembered sleep disturbances in the ICU - among these sleep-disturbed patients, $43 \%$ still declared a decreased long-term quality of sleep. In addition, $30 \%$ of patients reported a worse quality of sleep after their ICU stay than before admission. No relation was found when we assessed the possible effects of ICU variables on sleeping patterns during critical illness.
Sleep disturbances were described in one-half of the patients during their ICU stay. We noticed that one-third of the group declared having a poorer quality of sleep a long time after their discharge from the ICU. Our results differ from those of another study where the prevalence of self-reported quality of sleep did not change from the pre-ICU period to the post-ICU period and where the results did not incriminate the ICU stay in a long-term worse quality of sleep but did incriminate concurrent diseases [5]. The high self-reported prevalence of sleep disturbances, however, reveals the necessity for systematic protocols to improve sleep quality in the ICU.

Abbreviations

ICU, intensive care unit.

Competing interests

The authors declare that they have no competing interests.

Published: 7 March 2011

\section{References}

1. Friese RS: Sleep and recovery from critical illness and injury: a review of theory, current practice, and future directions. Crit Care Med 2008 36:697-705.

2. Simini B: Patients' perceptions of intensive care. Lancet 1999, 354:571-572.

3. Granja C, Lopes A, Moreira S, Dias C, Costa-Pereira A, Carneiro A; JMIP Study Group: Patients' recollections of experiences in the intensive care unit may affect their quality of life. Crit Care 2005, 9:R96-R109.

4. Partinen M, Gislason T: Basic Nordic Sleep Questionnaire (BNSQ): a quantitative measure of subjective sleep complaints. J Sleep Res 1995, 4:150-155.

5. Orwelius L, Nordlund A, Nordlund P, Edéll-Gustafsson U, Sjöberg F: Prevalence of sleep disturbances and long-term reduced health-related quality of life after critical care: a prospective multicenter cohort study. Crit Care 2008, 12:R97.

doi:10.1186/cc10042

Cite this article as: Franck $L$, et al.: How did you sleep in the ICU? Critical Care 2011, 15:408. 